\title{
Incidence and risk factors for venous thrombosis among patients with inflammatory bowel disease in China: a multicenter retrospective study
}

\author{
Jing Liu ${ }^{1,2}$, Xiang Gao ${ }^{3}$, Ye Chen ${ }^{4}$, Qiao $\mathrm{Mei}^{5}$, Liangru Zhu ${ }^{6}$, Jiaming Qian ${ }^{7}$, Pinjin $\mathrm{Hu}^{3}$, Qian Cao ${ }^{1}$ \\ ${ }^{1}$ Inflammatory Bowel Disease Center, Sir Run Run Shaw Hospital, Zhejiang University School of Medicine, Hangzhou; ${ }^{2}$ Department of Internal \\ Medicine, Peking Union Medical College Hospital, Beijing, ${ }^{3}$ Department of Gastroenterology, The Sixth Affiliated Hospital of Sun Yat-sen \\ University, Guangzhou; ${ }^{4}$ Department of Gastroenterology, Nanfang Hospital of Southern Medical University, Guangzhou; ${ }^{5}$ Department of \\ Gastroenterology, The First Affiliated Hospital of Anhui Medical University, Hefei; ${ }^{6}$ Department of Gastroenterology, Union Hospital, Tongji \\ Medical College, Huazhong University of Science and Technology, Wuhan; ${ }^{7}$ Department of Gastroenterology, Peking Union Medical College \\ Hospital, Beijing, China
}

Background/Aims: Risk of venous thrombosis is increased in patients with inflammatory bowel disease (IBD); data on Asian IBD patients is limited and status quo of thrombosis screening and prophylaxis are unknown. Therefore, we aimed to investigate the incidence, screening, prophylaxis, and risk factors for venous thrombosis among Asian IBD patients. Methods: Medical files of patients with Crohn's disease (CD) and ulcerative colitis (UC) from 17 hospitals across China between 2011 and 2016 were reviewed for venous thrombosis, use of screening and prophylaxis. A case-control study was performed among hospitalized patients with venous thrombosis and their age-, sex-matched IBD controls hospitalized around the same period; disease characteristics and known provoking factors of venous thrombosis were recorded. Risk factors were analyzed in both univariate and logistic regression analyses. Results: A total of 8,459 IBD patients were followed for 12,373 person-year. Forty-six patients (0.54\%) had venous thrombosis, yielding an incidence of 37.18 per 10,000 person-year. Incidence increased with age, especially among CD. Less than $20 \%$ of patients received screening tests and 35 patients $(0.41 \%)$ received prophylaxis. Severe disease flare was an independent risk factor for venous thrombosis (odds ratio [95\% confidence interval]: CD, 9.342 [1.81348.137]; UC, 5.198 [1.268-21.305]); past use of steroids and extensive involvement were 2 additional risk factors in CD and UC, respectively. Conclusions: Incidence of venous thrombosis in China was 37.18 per 10,000 person-year (0.54\%). Use of screening and prophylaxis were rare. Severe disease flare was an independent risk factor for thrombosis among hospitalized patients. (Intest Res 2021;19:313-322)

Key Words: Inflammatory bowel disease; Venous thrombosis; Pulmonary embolism

\section{INTRODUCTION}

Inflammatory bowel disease (IBD) is a risk factor for venous thrombosis, including deep venous thrombosis of lower extremities (DVT), pulmonary embolism (PE), and thrombosis

Received March 17, 2020. Revised September 3, 2020.

Accepted September 7, 2020.

Correspondence to Qian Cao, Inflammatory Bowel Disease Center, Sir Run

Run Shaw Hospital, Zhejiang University School of Medicine, No. 3, East

Qingchun Road, Hangzhou 310016, China. Tel: +86-13588706896,

Fax: +86-571-86044817, E-mail: caoq@zju.edu.cn of other veins such as intra-abdominal venous thrombosis. Patients with IBD, including Crohn's disease (CD) and ulcerative colitis (UC), have an average of 2- to 4-fold risk for venous thrombosis compared to the healthy population. ${ }^{1-4} \mathrm{Ve}-$ nous thrombosis results in an increased mortality rate, especially in patients who developed PE., The development of venous thrombosis among IBD patients is a multifactorial process that follow the triad of Virchow. ${ }^{7}$ Active inflammation further increased such risk, and therefore thromboprophylaxis has been recommended for IBD patients with high risk, in- 
cluding hospitalized IBD patients during disease flares. ${ }^{2}$

Studies regarding venous thrombosis among IBD patients mainly come from the Western population. Incidence of venous thrombosis varied across different regions, and for Asian population, it was reported to be lower compared to the West. ${ }^{8}$ Though the incidence of IBD in the Asian-Pacific region kept rising over the last decade, ${ }^{9}$ venous thrombosis still remains under-recognized in clinical practice in Asia. ${ }^{10}$ In recent years, 2 population-based studies investigated venous thrombosis among Asian IBD patients and discovered a relatively lower incidence compared to the Western IBD population. ${ }^{11,12}$ Similar epidemiology studies involving large populations are still scarce, and status quo of venous thrombosis screening and prophylaxis among Asian IBD patients remains unknown. Therefore, we aimed to investigate the incidence, status quo of screening and prophylaxis for venous thrombosis among Chinese IBD patients, and also to identify risk factors for venous thrombosis.

\section{METHODS}

\section{Study Population and Variables}

We performed a retrospective analysis of all patients with either CD or UC from 17 tertiary referral hospitals in China between January 2011 and June 2016. Both regular follow-up patients diagnosed with IBD prior to the starting date of the current study and newly-diagnosed patients during the study timeframe were included. Data were obtained by manual reviews of medical documents from each participating hospital. Standardized questionnaires were used for data collection. All questionnaires were completed by designated researchers from the participating hospitals, and then collected by one principal researcher responsible for data curation and subsequent analysis. Demographic features including age and sex, type of IBD, both disease and symptom duration of IBD were recorded. Thrombosis screening, use of prophylaxis, diagnosis and outcomes of venous thrombosis events were also recorded.

A case-control analysis was performed to investigate risk factors for venous thrombosis among hospitalized patients. Hospitalized IBD patients with venous thrombosis were 1:3 matched by sex and age ( \pm 3 years) to IBD patients without venous thrombosis who were hospitalized around similar period. Time-dependent information was collected using the index time as reference. The index time referred to the time around venous thrombosis diagnosis for patients with venous thrombosis, or the time at inclusion for control group. Detailed disease history around the index time were reviewed, including body mass index, smoking status, central catheter insertion, history of IBD-related bowel resection, history of cancer, history of venous thrombosis prior to IBD diagnosis, comorbidities including history of heart failure, diabetes, myocardial infarction, and stroke. Known provoking events for venous thrombosis including surgery, cancer or pregnancy within 3 months of index time were also noted. ${ }^{4}$ IBD disease behaviors were defined according to the Montreal classification. ${ }^{13}$ IBD disease activity was assessed using Mayo score for UC and Crohn's Disease Activity Index (CDAI) score for CD. ${ }^{14,15}$ Mayo score of less than 2, or CDAI score of less than 150 were considered disease remission, Mayo score between 3 and 5, or CDAI score between 150 and 220 were considered mildly-active disease, Mayo score between 6 and 10, or CDAI score between 221 and 450 were considered moderately-active disease, Mayo score of more than 11, or CDAI score of more than 450 were considered severely-active disease. IBD-related medication was also noted; past medication referred to treatment received beyond 1 month before the index time, and only medication used for longer than 3 months were included; current medication referred to treatment used within 1 month around index time. Exclusion criteria: (1) incomplete demographic information; (2) unconfirmed diagnosis of IBD according to current guideline; or (3) unknown time for followup or patients with only one clinic visit.

\section{Definition of Venous Thrombosis}

Venous thrombosis refers to thrombosis in the venous system or PE, the former included and not confined to DVT, thrombosis of upper extremities, and intra-abdominal venous thrombosis. Diagnosis of venous thrombosis should be made based on direct evidence from ultrasound, computed tomography or pulmonary angiography. Only events of venous thrombosis that occurred during the study timeframe were counted.

\section{Statistical Analysis}

Kolmogorov-Smirnov test was used to test for normality of data. Continuous variables with normal distribution were presented with mean \pm standard deviations; non-normal variables were reported as medium with interquartile ranges (IQRs). Mean of 2 continuous normally distributed variables were compared using independent $t$-test; non-normally distributed continuous variables were compared using Mann-Whitney $U$ test. Categorical variables were presented as percentages and compared using chi-square analysis. Hazard ratio was calcu- 
lated using Cox-proportional regression analysis. Fisher exact test was used when sample size was under 5 . All variables with $P<0.1$ in the univariate analysis were entered in a stepwise manner into the logistic regression. A $P$-value of $<0.05$ was considered statistically significant. All analysis was conducted using SPSS software version 22 (IBM Corp., Armonk, NY, USA).

\section{Ethical Statement}

This study was approved by Institutional Review Board of the Sir Run Run Shaw Hospital (IRB No. 20200721-33). The informed consent was waived.

\section{RESULTS}

\section{Baseline Characteristics and Incidence of Venous Thrombosis}

A total of 8,459 patients were eventually included, median time of follow-up was 9.6 months (IQR, 1.0-17.8 months). Forty-six patients $(0.54 \%)$ were identified as having venous thrombosis, yielding an incidence of 37.18 per 10,000 personyear (total follow-up of 12,373 person-year) for venous thrombosis. Demographic characteristics of patients with and without venous thrombosis are shown in Table 1. Patients with venous thrombosis were older compared to control. Sex, type of IBD, disease and symptom duration did not differ between patients with and without venous thrombosis.

Among all types of venous thrombosis, incidence of DVT was highest $(0.33 \%, 22.63$ per 10,000 person-year $)$, and $\mathrm{PE}$

Table 1. Baseline Characteristics of All Patients

\begin{tabular}{lccc}
\hline Variable & $\begin{array}{c}\text { With } \\
\text { thrombosis } \\
(\mathrm{n}=46)\end{array}$ & $\begin{array}{c}\text { Without } \\
\text { thrombosis } \\
(\mathrm{n}=8,413)\end{array}$ & $P$-value ${ }^{\mathrm{a}}$ \\
\hline Male sex & $26(56.5)$ & $5,282(62.8)$ & 0.445 \\
\hline Age (yr) & $46.3 \pm 15.7$ & $39.9 \pm 15.0$ & 0.004 \\
$<40$ & $17(37.0)$ & $4,556(54.2)$ & \\
$40-59$ & $18(39.1)$ & $2,873(34.1)$ & \\
$\geq 60$ & $11(23.9)$ & $984(11.7)$ & \\
\hline Type of IBD (CD) & $20(43.5)$ & $4,102(48.8)$ & 0.555 \\
Disease duration (mo) & $39.1(5.3-58.5)$ & $21.6(4.0-51.7)$ & 0.123 \\
Symptom duration (mo) & $53.4(20.5-111.8)$ & $48.7(22.1-81.2)$ & 0.465 \\
\hline
\end{tabular}

Values are presented as number (\%), mean \pm standard deviation, or median (interquartile range).

${ }^{a} P<0.05$, compared to patients without venous thrombosis

IBD, inflammatory bowel disease; CD, Crohn's disease. was lowest $(0.08 \%, 5.66$ per 10,000 person-year). The combined incidence of DVT and PE was 28.29 per 10,000 personyear. Incidence of venous thrombosis increased with age; such trend was most significant for DVT, and for patients with $\mathrm{CD}$ in particular. Comparing patients over 60 years old to patients under 40 years old, the hazard ratio for venous thrombosis and DVT were 2.776 and 3.343, respectively, which increased to 3.779 and 4.738 for patients with CD in particular and became insignificant for patients with UC. Risk of thrombosis other than DVT and PE did not differ among different age groups (Table 2).

\section{Screening, Prophylaxis, Treatment and Outcome for Venous Thrombosis}

A total of 1,633 patients (19.3\%) received D-dimer tests, 201 patients $(2.38 \%)$ received ultrasound screening, and 24 patients $(0.28 \%)$ received computed tomography scan for screening. Thirty-five patients $(0.41 \%)$ received prophylaxis including warfarin, heparin or low molecular weight heparin and none of these patients developed venous thrombosis. Among the 46 patients diagnosed with venous thrombosis, 28 patients developed DVT without progression to PE, 7 patients developed $\mathrm{PE}$, and 11 patients had venous thrombosis of other veins (Table 3). Thirty-five patients (76\%) reported symptoms in accordance with the affected veins, including swollen of the affected limbs, chest pain, or abdominal pain; 11 patients did not report any symptoms. Six out of 46 patients were diagnosed as outpatients and no details for treatment and outcome were recorded. Among the 40 patients being hospitalized, the median time of hospitalization was 27 days (IQR, 14-28 days). Duration of hospitalization was longer for patients over 60 years compared to patients under 60 years old ( 23 days [IQR, $12-35]$ vs. 39 days [IQR, 30-44], $P=0.033$ ). No differences in hospitalization duration were observed between $\mathrm{CD}$ and UC (31 days [IQR, 15-39] vs. 23 days [IQR, 13-38], $P=0.632$ ), male and female (21 days [IQR, 11-35] vs. 34 days [IQR, 19-39], $P=0.133$ ), or between DVT and PE (31 days [IQR, 15-39] vs. 34 days [IQR, 9-94], $P=0.971)$. Six patients lost follow-up after discharge, 32 patients had complete recovery from venous thrombosis, 1 patient progressed to pulmonary hypertension, and 1 patient with CD died due to $\mathrm{PE}$.

\section{Risk Factors Associated with Venous Thrombosis among Hospitalized Patients}

Hospitalized patients with venous thrombosis were matched to patients without venous thrombosis (Tables 4,5 ). None of 


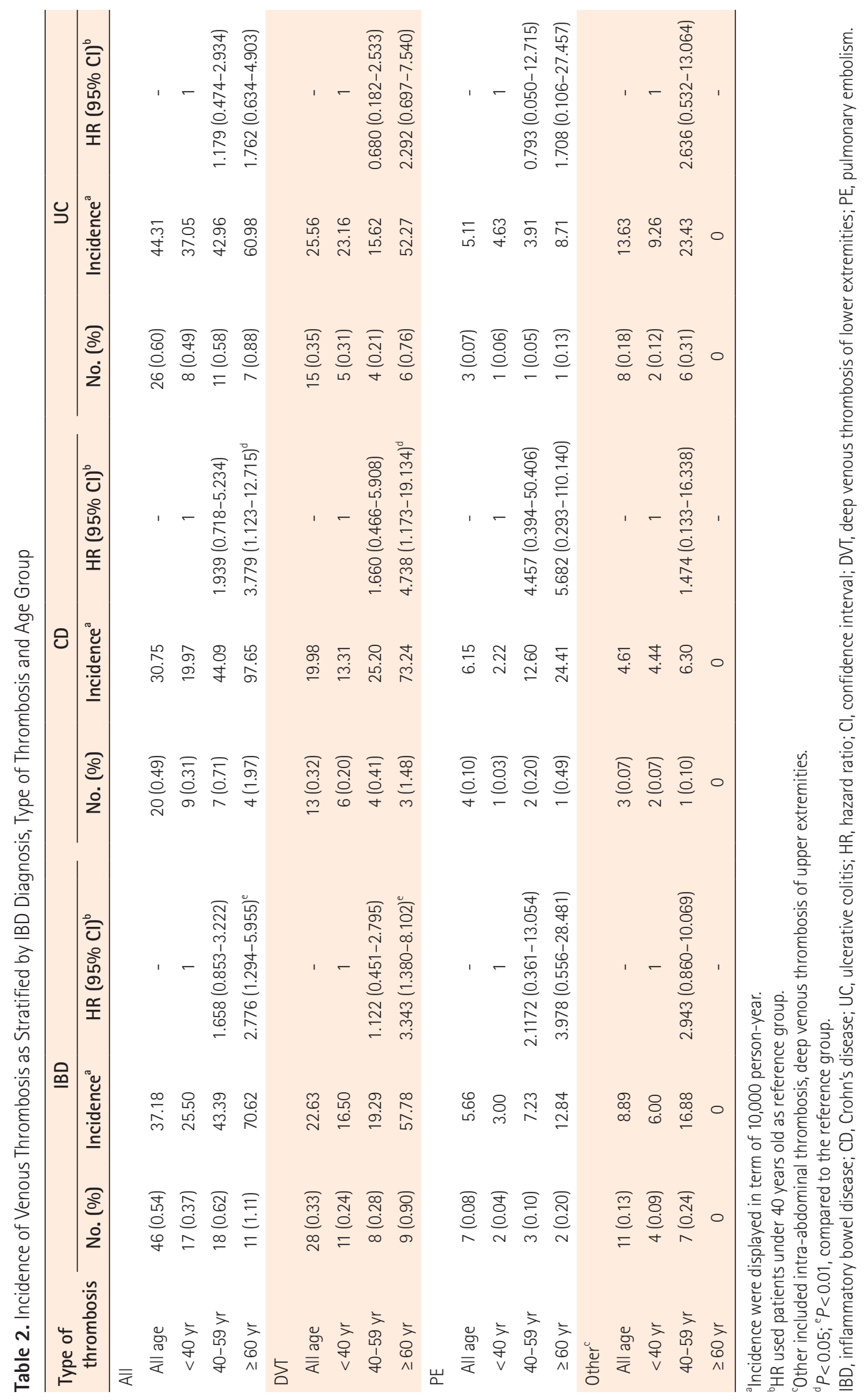


Table 3. Location of Venous Thrombosis

\begin{tabular}{llc}
\hline $\begin{array}{c}\text { Type of } \\
\text { thrombosis }\end{array}$ & \multicolumn{1}{c}{ Location } & $\begin{array}{c}\text { No. of } \\
\text { patients }\end{array}$ \\
\hline DVT $(n=28)$ & Lower limbs & 26 \\
& Lower limbs + inferior vena cava/portal vein & 2 \\
PE $(n=7)$ & Pulmonary artery + lower limbs & 6 \\
& Pulmonary artery + upper limbs & 1 \\
Others $(n=11)$ & Portal vein & 3 \\
& Mesenteric vein & 1 \\
& Splenic vein & 1 \\
& Inferior vena cava & 1 \\
& Portal vein + mesenteric vein & 3 \\
& Upper limb + jugular vein & 1 \\
& Upper limb + subclavian vein & 1 \\
\hline
\end{tabular}

DVT, deep venous thrombosis of lower extremities; PE, pulmonary embolism.

patients reported venous thrombosis prior to the diagnosis of IBD, use of oral contraceptive, or cancer within 3 months of venous thrombosis diagnosis. For CD patients, neither disease location nor disease behavior differed between patients with and without venous thrombosis, whereas for UC patients, those with venous thrombosis were more likely to have pancolitis.

Most of patients had disease flares regardless of venous thrombosis diagnosis (Fig. 1), and the 2 groups did not differ in the proportion of patients in remission. Patients with venous thrombosis were more likely to have severe disease flares, and disease activity scores were generally higher among patients with venous thrombosis compared to control (CDAI score for CD: $325.0 \pm 148.1$ vs. $203.4 \pm 65.8, P<0.01$; Mayo score for UC: 11 [9-12] vs. 7 [6-9], $P<0.01)$. In both the univariate and multivariate logistic regression analysis (Table 6), severe disease flare was an independent risk factor for venous thrombosis, regardless of the underlying IBD diagnosis. For CD patients, past use of steroids was an additional independent risk factor for venous thrombosis; the rest variables that were significant in the univariate analysis became insignificant, including history of cancer $(P=0.999)$, comorbidity $(P=0.999)$, past use of steroids $(P=0.597)$, and past use of immunosuppressants $(P=0.702)$. For UC patients, extensive disease involvement was an additional independent risk factors for venous thrombosis; the rest variables that were significant in the univariate analysis became insignificant, including central catheter insertions $(P=0.285)$, history of IBD-related bowel re-
Table 4. Univariate Analysis of Risk Factors for Venous Thrombosis among CD Patients

\begin{tabular}{|c|c|c|c|}
\hline Variable & $\begin{array}{l}\text { Thrombosis } \\
(n=16)\end{array}$ & $\begin{array}{l}\text { Control } \\
(n=48)\end{array}$ & $P$-value \\
\hline Age (yr) & $41.1 \pm 17.5$ & $37.7 \pm 12.8$ & 0.419 \\
\hline Male sex & $11(69)$ & 33 (69) & 1.000 \\
\hline BMI $\left(\mathrm{kg} / \mathrm{m}^{2}\right)$ & $18.0 \pm 3.1$ & $18.8 \pm 3.0$ & 0.406 \\
\hline Surgery within 3 months & $2(13)$ & $4(9)$ & 0.348 \\
\hline Pregnancy within 3 months & 0 & 0 & 1.000 \\
\hline History of cancer & $2(13)$ & $1(2)$ & 0.061 \\
\hline Active smoker & $1(6)$ & $7(15)$ & 0.667 \\
\hline Central catheter insertion & $1(6)$ & 0 & 0.267 \\
\hline $\begin{array}{l}\text { History of IBD-related } \\
\text { bowel resection }\end{array}$ & $8(50)$ & $13(27)$ & 0.126 \\
\hline Comorbidity & $5(31)$ & $3(6)$ & 0.019 \\
\hline Disease location & & & 0.257 \\
\hline Terminal ileum & $6(37)$ & $20(42)$ & \\
\hline Colon & 0 & $6(13)$ & \\
\hline Ileocolon & $10(63)$ & $22(46)$ & \\
\hline $\begin{array}{l}\text { Upper gastrointestinal tract } \\
\text { involvement }\end{array}$ & $1(6)$ & $6(13)$ & 0.669 \\
\hline Disease behavior & & & 0.150 \\
\hline Inflammatory & $4(25)$ & $22(46)$ & \\
\hline Stricturing & $7(44)$ & $17(35)$ & \\
\hline Penetrating & $5(31)$ & $6(13)$ & \\
\hline Perianal disease & $1(6)$ & $9(19)$ & 0.430 \\
\hline Disease remission & $3(19)$ & $21(44)$ & 0.342 \\
\hline Severe disease flare & $7(44)$ & $4(8)$ & 0.003 \\
\hline \multicolumn{4}{|l|}{ Past IBD medication } \\
\hline Aminosalicylic acid & $9(56)$ & $27(56)$ & 1.000 \\
\hline Steroids & $11(69)$ & $18(38)$ & 0.043 \\
\hline Immunosuppressants & $10(63)$ & $18(38)$ & 0.092 \\
\hline Biologics & $4(25)$ & $12(25)$ & 1.000 \\
\hline \multicolumn{4}{|l|}{ Current IBD medication } \\
\hline Aminosalicylic acid & 0 & $6(13)$ & 0.321 \\
\hline Steroids & $4(27)$ & $4(8)$ & 0.084 \\
\hline Immunosuppressants & $5(33)$ & $9(19)$ & 0.274 \\
\hline Biologics & $1(7)$ & $6(13)$ & 1.000 \\
\hline
\end{tabular}

Values are presented as mean \pm standard deviation or number (\%). Body mass index (BMI) were missing for 2 Crohn's disease (CD) patients with venous thrombosis; history of surgery within 3 months were missing for $1 \mathrm{CD}$ patients with venous thrombosis and 2 control CD patients; history of cancer were missing for $1 \mathrm{CD}$ patients with venous thrombosis and 1 control CD patients; central catheter insertion were missing for 4 control CD patients; disease behavior were missing for 3 control CD patients; current use of inflammatory bowel disease (IBD) medication were missing for $1 \mathrm{CD}$ patients with venous thrombosis.

section $(P=0.204)$, past use of aminosalicylic acid $(P=0.405)$, and current use of steroids $(P=0.986)$. 
Table 5. Univariate Analysis of Risk Factors for Venous Thrombosis among UC Patients

\begin{tabular}{|c|c|c|c|}
\hline Variable & $\begin{array}{l}\text { Thrombosis } \\
(n=24)\end{array}$ & $\begin{array}{l}\text { Control } \\
(n=72)\end{array}$ & $P$-value \\
\hline Age (yr) & $47.2 \pm 14.7$ & $46.0 \pm 16.6$ & 0.760 \\
\hline Male sex & $12(50)$ & $36(50)$ & 1.000 \\
\hline BMI $\left(\mathrm{kg} / \mathrm{m}^{2}\right)$ & $20.9 \pm 3.5$ & $21.2 \pm 3.3$ & 0.783 \\
\hline Surgery within 3 months & $4(17)$ & $3(4)$ & 0.167 \\
\hline Pregnancy within 3 months & $1(4)$ & 0 & 0.253 \\
\hline History of cancer & 0 & $1(1)$ & 1.000 \\
\hline Active smoker & $4(17)$ & $11(15)$ & 1.000 \\
\hline Central catheter insertion & $3(13)$ & 0 & 0.020 \\
\hline $\begin{array}{l}\text { History of IBD-related } \\
\text { bowel resection }\end{array}$ & $3(13)$ & $2(3)$ & 0.098 \\
\hline Comorbidity & $4(17)$ & $11(15)$ & 1.000 \\
\hline Disease location & & & 0.008 \\
\hline Proctitis & $1(4)$ & $17(23)$ & \\
\hline Left-sided colitis & $2(8)$ & $19(26)$ & \\
\hline Pancolitis & $21(88)$ & $36(49)$ & \\
\hline Disease remission & 0 & $2(3)$ & 1.000 \\
\hline Severe disease flare & $10(48)$ & $8(11)$ & 0.001 \\
\hline \multicolumn{4}{|l|}{ Past IBD medication } \\
\hline Aminosalicylic acid & $12(52)$ & $62(86)$ & 0.001 \\
\hline Steroids & $12(52)$ & $30(42)$ & 0.471 \\
\hline Immunosuppressants & $2(9)$ & $6(8)$ & 1.000 \\
\hline Biologics & $1(5)$ & $1(1)$ & 0.428 \\
\hline \multicolumn{4}{|l|}{ Current IBD medication } \\
\hline Aminosalicylic acid & $11(48)$ & $36(50)$ & 0.815 \\
\hline Steroids & $13(57)$ & $13(18)$ & 0.001 \\
\hline Immunosuppressants & $2(9)$ & $4(6)$ & 0.630 \\
\hline Biologics & 0 & 0 & 1.000 \\
\hline
\end{tabular}

Values are presented as mean \pm standard deviation or number (\%). Body mass index (BMI) were missing for 5 ulcerative colitis (UC) patients with venous thrombosis and 9 control UC patients; history of surgery within 3 months were missing for $1 \mathrm{UC}$ patients with venous thrombosis and 1 control UC patients; central catheter insertion were missing for 11 control UC patients; disease severity were not assessed for 3 UC patients with venous thrombosis; past use of inflammatory bowel disease (IBD) medication were missing for 1 UC patients with venous thrombosis; current use of IBD medication were missing for 2 UC patients with venous thrombosis.

\section{DISCUSSION}

In this multicenter hospital-based study we reported an incidence of 37.18 per 10,000 person-year $(0.54 \%)$ for venous thrombosis among Chinese IBD patients. Risk for venous thrombosis was highest among older patients, especially patients with CD. Thrombosis screening was not routinely per-

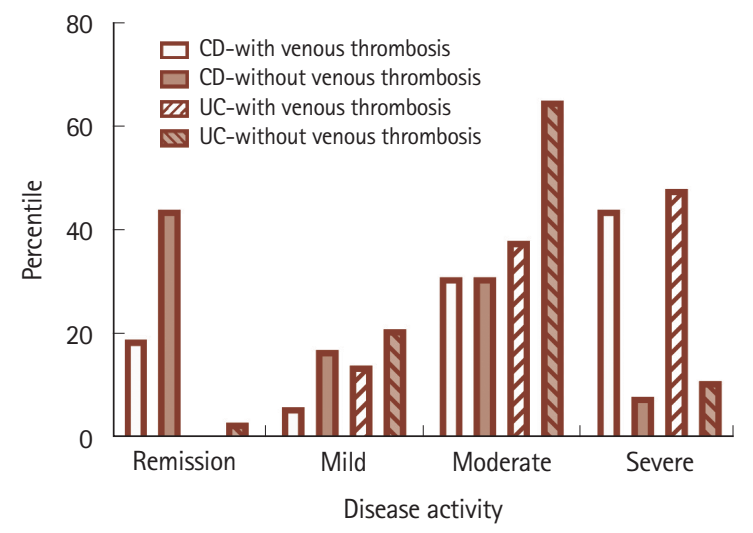

Fig. 1. Disease activity for Crohn's disease (CD), ulcerative colitis (UC) patients with and without venous thrombosis. Disease activity was assessed using Mayo score for UC and Crohn's Disease Activity Index (CDAl) score for CD. Mayo score of $\leq 2$, or CDAl score $<150$ were considered disease remission, Mayo score between 3 and 5, or CDAl score between 150 and 220 were considered mildly-active disease, Mayo score between 6 and 10, or CDAl score between 221 and 450 were considered moderately-active disease, Mayo score of more than 11, or CDAl score of more than 450 were considered as severe disease activity.

Table 6. Multivariate Logistic Regression of Risk Factor for Venous Thrombosis among IBD Patients

\begin{tabular}{lcc}
\hline Variable & OR (95\% Cl) & $P$-value \\
\hline CD & & \\
Severe disease flare (yes vs. no) & $9.342(1.813-48.137)$ & 0.008 \\
Past use of steroids (yes vs. no) & $5.672(1.331-24.165)$ & 0.019 \\
UC & & \\
Extensive involvement (yes vs. no) & $5.810(1.123-30.059)$ & 0.036 \\
Severe disease flare (yes vs. no) & $5.198(1.268-21.305)$ & 0.022 \\
\hline
\end{tabular}

IBD, inflammatory bowel disease; $C D$, Crohn's disease; UC, ulcerative colitis; $\mathrm{OR}$, odds ratio; $\mathrm{Cl}$, confidence interval.

formed and thromboprophylaxis was rarely used; around $20 \%$ of the patients received tests related to thrombosis screening and less than $1 \%$ of the patients received prophylaxis. Severe disease flare was an independent risk factor for venous thrombosis among hospitalized IBD patients.

IBD is an independent risk factor for venous thrombosis, and IBD patients had 2- to 4-fold risk of venous thrombosis compared to the general population. ${ }^{3,4,16}$ Combined incidence for DVT and PE among IBD in Denmark and Canada ranged from 24 to 45.6 per 10,000 person-years. ${ }^{1,3,4}$ Asian population had lower incidence of venous thrombosis compared to the Western population. ${ }^{8}$ In recent years, 2 population-based 
studies demonstrated 2-fold risks for venous thrombosis among IBD patients in East-Asia, similar to the Western population. ${ }^{11,12}$ However, the actual incidence of venous thrombosis in these 2 studies ranged from 6 to 9.81 per 10,000 personyears, ${ }^{11,12,17}$ with lower proportion of patients $(0.79 \%-1.31 \%)$ with venous thrombosis compared to the Western population $(3 \%-4 \%)$. The proportion of patients with venous thrombosis in the current study was similar to previous reports from Asia. Given such low proportion of patients with venous thrombosis, the high incidence was more likely to be overrated due to the relatively short follow-up time of the recruited patients, instead of selection bias as a result of potentially more severe disease presentations among patients that were enrolled from tertiary hospitals in the current study.

In addition to the incidence of venous thrombosis, we also reported that thrombosis screening and use of prophylaxis were relatively rare among IBD patients in Asia. Thromboprophylaxis was not considered a regular practice for over half of IBD physicians in Asia. ${ }^{10}$ The actual use of thromboprophylaxis might be lower; we discovered that less than $1 \%$ of patients received thromboprophylaxis, which is similar to a report from Singapore in which none of the 152 hospitalized IBD patients received thromboprophylaxis. ${ }^{17}$ Lower incidence of venous thrombosis results in less commonly practiced thromboprophylaxis compared to the West; however, close attention is needed for patients with high risk. ${ }^{18}$ For the general population, older patients are among the high-risk groups for venous thrombosis. ${ }^{8}$ In the current study, we discovered that risk for venous thrombosis also increased with age, and hospitalizations were longer among older patients. As aging becomes prevalent among the growing IBD population, venous thrombosis could be an emerging complication causing considerable mortality and impaired quality-adjusted life-years. ${ }^{6}$ Therefore, standardized protocols for thromboprophylaxis for IBD patients in Asia are necessary, especially for patients with additional risks for venous thrombosis. ${ }^{18}$ Many factors influence the cost-effectiveness of thromboprophylaxis, including incidence of venous thrombosis, the benefits of prophylaxis against risk of bleeding in different patient subgroups, and also socio-economic factors such as cost and compliance. ${ }^{19,20}$ For patients in Asia, due to lower incidence of venous thrombosis in general and different socio-economic backgrounds, precise risk stratification models are needed. This would require incidence and risk factor studies among different patient subgroups, and also investigations into risk and benefits of thromboprophylaxis among Asian IBD patients.
Though thromboprophylaxis may not be a routine practice in the context of low incidence of venous thrombosis, screening and close monitoring are still necessary since IBD patients had 2-fold risk for venous thrombosis. ${ }^{11,12}$ However, we discovered that use of thrombosis screening was relatively rare. Two previous studies from Japan showed much higher rates, ranging between $3.6 \%$ to $27.2 \%$, for venous thrombosis with the use of proactive screening. Surprisingly, more than $50 \%$ of the reported thrombotic events were asymptomatic. ${ }^{21,22}$ Therefore, proactive screening could be useful for early identification of thrombosis; this is especially important for thrombosis with atypical presentation such as intra-abdominal thrombosis. In our study, more patients developed intra-abdominal venous thrombosis than PE, and the reported symptoms were mostly nonspecific such as abdominal pain. Intra-abdominal thrombosis are common, especially for UC patients after surgery; recanalization is usually required to prevent deadly bowel ischemia and recurrent thrombosis. ${ }^{23}$ Symptoms of intra-abdominal thrombosis are highly variable and could be confounded by IBD disease flare per se, causing delay in diagnosis and treatment. Therefore, screening for thrombotic events is crucial as it may avoid treatment delay if venous thrombosis truly develops.

Evidence regarding the appropriate choice of screening methods for IBD patients in Asia is limited. In the current study, the modality of screening was mainly D-dimer tests, with less than $3 \%$ of patients receiving ultrasound screening or computed tomography scans. A recent study showed elevated D-dimer at admission was highly predictive for venous thrombosis and therefore D-dimer test might be a useful screening tool. ${ }^{24}$ However, another study suggested that the utility of D-dimer tests as screening methods could be limited, since elevated D-dimer is common in inflammatory diseases. Therefore, though D-dimer test is economical for initial screening, direct imaging study might be more appropriate for patients with high risks. ${ }^{25}$ Further investigation into cost-effectiveness of screening methods are needed, especially for patients with high risk, such as patients during disease flares and patients undergoing surgery.

Several risk factors for venous thrombosis among hospitalized IBD patients have been previously reported, including disease flares, extensive disease, surgeries, use of steroids, and even hospitalization per se. ${ }^{6,18,26-30}$ Similar to those previously reported, we discovered that severe disease flare was an independent risk factor for venous thrombosis. Elevated risks of venous thrombosis were reported among many autoimmune 
diseases as a result of inflammation; ${ }^{7,31,32}$ IBD patients during disease flares had 8-fold risks of venous thrombosis., ${ }^{1,2,16,33}$ Most of patients in the our case-control study had active disease, possibly due to selection bias since patients were usually hospitalized due to flares. However, we showed that a more severely-active disease still exposed patients to higher risk for venous thrombosis. In addition, extensive gastrointestinal involvement exposed patients to higher risk of venous thrombosis, which was consistent with previous discoveries that pancolitis was associated with increased risk of venous thrombosis among UC patients. ${ }^{12,16,28,30}$ These preliminary findings provided identifiable factors for risk stratification among hospitalized patients. Patients with these risk factors might be candidates to more proactive screening and prophylaxis.

The current study has several limitations. First, unlike previous population-based studies, relative risk of venous thrombosis for IBD patients was not analyzed due to lack of health data registry of the Chinese population in general. Second, due to limited access to resources as a retrospective multicenter study, only patients from tertiary referral hospitals were included, causing potential selection bias; in addition, hospitalized and ambulatory patients were not separately assessed. Therefore, we were not able to directly compare incidence and risk factors of venous thrombosis among different patient groups. Lack of such analysis limited the generalizability of the results, and therefore results of this study should only be applied to patients from tertiary referral hospitals. Future studies investigating venous thrombosis risk among different subgroups, such as patients being recently discharged, or postoperative patients, would provide evidence for more precise risk stratification strategies.

In conclusion, this study included a large number of IBD patients from tertiary referral hospitals as a representative of the Asian population and demonstrated a relatively low rate of venous thrombosis compared to the Western population. Thrombosis screening was only performed in a small proportion of patients, and prophylaxis was rarely practiced. Severely-active disease was a risk factor for hospitalized patients. Based on these findings, we suggested that thromboprophylaxis should be administered with caution given the lower likelihood of venous thrombosis. We also recommended that thrombosis screening could be valuable in avoiding treatment delay, especially for patients at high risks. Lastly, future studies are needed to explore risk stratification strategies for IBD patients in Asia, and also to evaluate the cost-effectiveness of thromboprophylaxis.

\section{ADDITIONAL INFORMATION}

\section{Funding Source}

The authors received no financial support for the research, authorship, and/or publication of this article.

\section{Conflict of Interest}

Gao X and Qian J are editorial board members of the journal but were not involved in the peer reviewer selection, evaluation, or decision process of this article. No other potential conflicts of interest relevant to this article were reported.

\section{Author Contribution}

Conceptualization: all authors. Data curation: Liu J. Formal analysis: Liu J, Cao Q. Investigation: Liu J, Hu P, Cao Q. Methodology: Liu J, Zhu L, Hu P, Cao Q. Project administration: all authors. Resources: Gao X, Chen Y, Mei Q, Zhu L, Qian J, Hu P, Cao Q. Software: Liu J. Supervision: Gao X, Mei Q, Zhu L, Qian J, Hu P, Cao Q. Validation: Liu J. Visualization: Liu J. Writing original draft: Liu J. Writing - review \& editing: Liu J, Gao X, Chen Y, Hu P, Cao Q. Approval of final manuscript: all authors.

\section{Others}

We give our thanks to the following doctors for their help with data collection: Ying Han, Feng Tian, Hu Zhang, Xiaolan Zhang, Yinglei Miao, Xiaofeng Yu, Huaxiu Shi, Chengdang Wang, Yulan Liu, Xiaoyan Wang, Xuefeng Li, Chunxiao Chen, Xiaoqi Zhang, Lanxiang Zhu, Yufang Wang, Yan Zhang, Changqing Zheng, Meifang Huang, Hongjie Zhang, Wen Tang, Baisui Feng, Xiaowei Liu, Huixiang Yang, Xiaocang Cao, Qiaomin Wang, Xiaoping Lv, Feihu Bai, Zhihua Ran, Qifang Zhang, Junxia Li, Xinxin Zhou, Xinyan Yang, Qingfan Yang, Junrong Chen, Juan Luo, Ning Chen, Xiaolei Zhao, Wei Han, Di Guo, Yonghua Shen, Hui Li, Xia Zhao, Jiaming Zhang, Chuan Chen, Min Zhu, Yahui Guo, Ping Jiang, Yan Zhuang, Siqi Wang, Li Tian, Yanjun Chen, Jun Shen, Hong Yang, Hailian Zhang, Jiaqin Xu, Feng Zhou, Lianjie Lin, Jingjing Ma, Guanghui Lian, Dandan Zhou, Yunquan Zheng, Jintong Chen, Xiwen Liu.

\section{ORCID}

Liu J

Gao X

Chen Y

Mei Q

Zhu L

Qian J https://orcid.org/0000-0002-8135-8893 https://orcid.org/0000-0001-5480-0781 https://orcid.org/0000-0002-7964-7899 https://orcid.org/0000-0002-0635-6564 https://orcid.org/0000-0002-7372-294X https://orcid.org/0000-0002-6252-7022 
Hu P

Cao Q

https://orcid.org/0000-0001-8603-4743

https://orcid.org/0000-0002-7427-6347

\section{REFERENCES}

1. Grainge MJ, West J, Card TR. Venous thromboembolism during active disease and remission in inflammatory bowel disease: a cohort study. Lancet 2010;375:657-663.

2. Nguyen GC, Bernstein CN, Bitton A, et al. Consensus statements on the risk, prevention, and treatment of venous thromboembolism in inflammatory bowel disease: Canadian Association of Gastroenterology. Gastroenterology 2014;146:835-848.

3. Bernstein CN, Blanchard JF, Houston DS, Wajda A. The incidence of deep venous thrombosis and pulmonary embolism among patients with inflammatory bowel disease: a population-based cohort study. Thromb Haemost 2001;85:430-434.

4. Kappelman MD, Horvath-Puho E, Sandler RS, et al. Thromboembolic risk among Danish children and adults with inflammatory bowel diseases: a population-based nationwide study. Gut 2011;60:937-943.

5. Jess T, Gamborg M, Munkholm P, Sørensen TI. Overall and cause-specific mortality in ulcerative colitis: meta-analysis of population-based inception cohort studies. Am J Gastroentero. 2007;102:609-617.

6. Andrade AR, Barros LL, Azevedo MF, et al. Risk of thrombosis and mortality in inflammatory bowel disease. Clin Transl Gastroenterol 2018;9:142.

7. Danese S, Papa A, Saibeni S, Repici A, Malesci A, Vecchi M. Inflammation and coagulation in inflammatory bowel disease: the clot thickens. Am J Gastroenterol 2007;102:174-186.

8. Raskob GE, Angchaisuksiri P, Blanco AN, et al. Thrombosis: a major contributor to global disease burden. Semin Thromb Hemost 2014;40:724-735.

9. Ng SC, Leung WK, Shi HY, et al. Epidemiology of inflammatory bowel disease from 1981 to 2014: results from a territorywide population-based registry in Hong Kong. Inflamm Bowel Dis 2016;22:1954-1960.

10. Song HK, Lee KM, Jung SA, et al. Quality of care in inflammatory bowel disease in Asia: the results of a multinational webbased survey in the 2(nd) Asian Organization of Crohn's and Colitis (AOCC) meeting in Seoul. Intest Res 2016;14:240-247.

11. Chung WS, Lin CL, Hsu WH, Kao CH. Inflammatory bowel disease increases the risks of deep vein thrombosis and pulmonary embolism in the hospitalized patients: a nationwide cohort study. Thromb Res 2015;135:492-496.

12. Weng MT, Park SH, Matsuoka K, et al. Incidence and risk fac- tor analysis of thromboembolic events in East Asian patients with inflammatory bowel disease, a multinational collaborative study. Inflamm Bowel Dis 2018;24:1791-1800.

13. Silverberg MS, Satsangi J, Ahmad T, et al. Toward an integrated clinical, molecular and serological classification of inflammatory bowel disease: report of a Working Party of the 2005 Montreal World Congress of Gastroenterology. Can J Gastroenterol 2005;19 Suppl A:5A-36A.

14. Schroeder KW, Tremaine WJ, Ilstrup DM. Coated oral 5-aminosalicylic acid therapy for mildly to moderately active ulcerative colitis: a randomized study. N Engl J Med 1987;317:1625-1629.

15. Best WR, Becktel JM, Singleton JW, Kern F Jr. Development of a Crohn's disease activity index. National Cooperative Crohn's Disease Study. Gastroenterology 1976;70:439-444.

16. Miehsler W, Reinisch W, Valic E, et al. Is inflammatory bowel disease an independent and disease specific risk factor for thromboembolism? Gut 2004;53:542-548.

17. Lin H, Lim WC. Venous thromboembolism in Asian patients with inflammatory bowel disease. J Crohns Colitis 2014;8 Suppl 1:S311-S312.

18. Weng MT, Tung CC, Wong JM, Wei SC. Should Asian inflammatory bowel disease patients need routine thromboprophylaxis? Intest Res 2018;16:312-314.

19. Lightner AL. Should surgical inflammatory bowel disease patients be given extended venous thromboembolic prophylaxis postoperatively? Inflamm Bowel Dis 2020;26:289-290.

20. Faye AS, Hung KW, Cheng K, et al. Minor hematochezia decreases use of venous thromboembolism prophylaxis in patients with inflammatory bowel disease. Inflamm Bowel Dis 2020;26:1394-1400.

21. Ando K, Fujiya M, Nomura Y, et al. The incidence and risk factors of venous thromboembolism in Japanese inpatients with inflammatory bowel disease: a retrospective cohort study. Intest Res 2018;16:416-425.

22. Ando K, Fujiya M, Nomura Y, et al. The incidence and risk factors of venous thromboembolism in patients with inflammatory bowel disease: a prospective multicenter cohort study. Digestion 2019;100:229-237.

23. Kayal M, Radcliffe M, Plietz M, et al. Portomesenteric venous thrombosis in patients undergoing surgery for medically refractory ulcerative colitis. Inflamm Bowel Dis 2020;26:283288.

24. Ohta Y, Arai M, Nakagawa T, et al. Comparison of a novel predictor of venous thromboembolic complications in inflammatory bowel disease with current predictors. J Gastroenterol Hepatol 2019;34:870-879. 
25. Bates SM, Greer IA, Middeldorp S, Veenstra DL, Prabulos AM, Vandvik PO. VTE, thrombophilia, antithrombotic therapy, and pregnancy: Antithrombotic Therapy and Prevention of Thrombosis, 9th ed: American College of Chest Physicians EvidenceBased Clinical Practice Guidelines. Chest 2012;141(2 Suppl): e691S-e736S.

26. Higgins PD, Skup M, Mulani PM, Lin J, Chao J. Increased risk of venous thromboembolic events with corticosteroid vs biologic therapy for inflammatory bowel disease. Clin Gastroenterol Hepatol 2015;13:316-321.

27. Koutroubakis IE. Therapy insight: vascular complications in patients with inflammatory bowel disease. Nat Clin Pract Gastroenterol Hepatol 2005;2:266-272.

28. Papay P, Miehsler W, Tilg H, et al. Clinical presentation of venous thromboembolism in inflammatory bowel disease. J Crohns Colitis 2013;7:723-729.

29. Scoville EA, Konijeti GG, Nguyen DD, Sauk J, Yajnik V, Anan- thakrishnan AN. Venous thromboembolism in patients with inflammatory bowel diseases: a case-control study of risk factors. Inflamm Bowel Dis 2014;20:631-636.

30. Zezos P, Kouklakis G, Saibil F. Inflammatory bowel disease and thromboembolism. World J Gastroenterol 2014;20:1386313878.

31. Tichelaar YI, Kluin-Nelemans HJ, Meijer K. Infections and inflammatory diseases as risk factors for venous thrombosis: a systematic review. Thromb Haemost 2012;107:827-837.

32. Zöller B, Li X, Sundquist J, Sundquist K. Risk of pulmonary embolism in patients with autoimmune disorders: a nationwide follow-up study from Sweden. Lancet 2012;379:244-249.

33. Wong T, Nightingale J, Winter M, Muller AF. Spontaneous venous thrombosis in inflammatory bowel disease: relevance of factor $\mathrm{V}$ Leiden and the prothrombin gene mutation. J Thromb Haemost 2003;1:1326-1328. 